\title{
Effect of The Body Water and The Body Composition, Calculated by Bioelectrical Impedance Analysis, On Contrast-Enhanced Dynamic Computed Tomography Images of The Liver.
}

Takanori Masuda ( $\nabla$ takanorimasuda@yahoo.co.jp )

Kawasaki University of Medical Welfare

Takeshi Nakaura

Kumamoto University

Yoshinori Funama

Kumamoto University

Tomoyasu Sato

Tsuchiya General Hospital

Keiko Arataki

Tsuchiya General Hospital

Takayuki Oku

Tsuchiya General Hospital

Shouko Masuda

kawamura Clinic

\section{Shinichi Arao}

Kawasaki University of Medical Welfare

Jyunichi Hiratsuka

Kawasaki University of Medical Welfare

Kazuo Awai

Hiroshima University

\section{Research Article}

Keywords: body composition, CE-DCT, BIA, HAP

Posted Date: July 24th, 2021

DOl: https://doi.org/10.21203/rs.3.rs-691821/v1

License: (c) (1) This work is licensed under a Creative Commons Attribution 4.0 International License. Read Full License 
Page 2/14 


\section{Abstract}

To improve its diagnosis on contrast-enhanced dynamic CT (CE-DCT) scans, contrast injection protocol must yield stable arterial contrast enhancement. To investigate how the body-water distribution and the body composition, calculated by bioelectrical impedance analysis (BIA), affect aortic and hepatic enhancement on CE-DCT scans. 236 patients with liver cirrhosis underwent CE-DCT before BIA. The CT number (in Hounsfield units, $\mathrm{HU}$ ) of the abdominal aorta at the celiac artery level on unenhanced scans and during the hepatic arterial phase (HAP) scans was recorded. And, the mean CT number of the hepatic parenchyma of both hepatic lobes at the celiac artery level on unenhanced and portal venous phase (PVP) scans was recorded. We calculated changes in the iodine dose per contrast enhancement $(\mathrm{mgl} / \mathrm{HU})$ (IDCE) to evaluate the effect of the patient age and of various constituents of the body composition by performing BIA. The IDCE of the abdominal aorta during HAP was $121.5 \pm 32.1 \mathrm{mgl} / \mathrm{HU}$; it was $698.7 \pm$ $211.1 \mathrm{mgl} / \mathrm{HU}$ in the hepatic parenchyma during the PVP. Among the parameters used in our BIA, the total body weight (TBW) was the most important factor affecting the IDCE of the liver abdominal aorta on CEDCT scans acquired during the HAP $(r=0.83)$. The TBW and the skeletal muscle index most strongly affected the IDCE of the hepatic parenchyma on CE-DCT scans obtained during the PVP $(r=0.69)$. The TBW had the strongest effect on contrast enhancement. The skeletal muscle index exhibited the strongest correlation with hepatic parenchymal contrast enhancement during the PVP.

\section{Introduction}

Contrast-enhanced dynamic CT (CE-DCT) facilitates the detection of hepatocellular carcinoma (HCC) ${ }^{1-4}$. To improve its diagnosis on CE-DCT scans, contrast enhancement must be at least 280 Hounsfield units $(\mathrm{HU})$ with vessel and $50 \mathrm{HU}$ with hepatic parenchyma ${ }^{5,6}$ and the contrast injection protocol must yield stable arterial contrast enhancement $1,7,8$.

As the patient age, sex, total body weight (TBW), height, cardiac output, and the presence of other diseases affect contrast enhancement under identical contrast injection protocols ${ }^{9,10}$, body size index such as the TBW, body mass index (BMI), body surface area (BSA), and the lean body weight (LBW) must be taken into account in patients subjected to CE-DCT $5,6,11-14$.

The human body contains water, protein, fat, and minerals. Because bioelectrical impedance analysis (BIA) applies frequencies ranging from $1 \mathrm{kHz}$ to $1 \mathrm{MHz}{ }^{15}$, it yields accurate results irrespective of the body type, -composition, and water distribution. Under the hypothesis that BIA would reveal additional factors affecting aortic- and hepatic parenchymal contrast enhancement, we investigated the effects of the water distribution and the body composition on aortic- and hepatic parenchymal enhancement on CEDCT scans.

\section{Methods}


This retrospective study was approved by our institutional review board (Tsuchiya General Hospital); informed patient consent was waived. We adhered to the tenets of the Declaration of Helsinki.

\section{Patients}

Between January 2019 and May 2020, 251 consecutive patients with suspected liver cirrhosis underwent CE-DCT. Excluded from subsequent BIA were 2 patients with a history of allergic reactions to iodinated contrast medium (CM) and 13 hemodialysis patients because contrast enhancement did not suffice for a precise evaluation ${ }^{9}$. The final study population consisted of 236 patients ( 143 men and 93 women). Their age ranged from 30 to 89 years.

\section{$\mathrm{BIA}$}

With the patient standing upright, BIA, performed with InBody770 (InBody Japan Inc., Tokyo, Japan), was carried out an average of one hour before CT scanning. The patients' age, height, and sex, obtained from our electronic medical record system, were entered for BIA. The PA was measured at $50 \mathrm{kHz}$; the estimated segmental body composition was obtained at multiple frequencies. The direct segmental multi-frequency bioimpedance analysis method was applied using a tetra-, polar 8-point tactile electrode system; 30 impedance measurements were obtained using 6 frequencies.

For body composition determination, BIA considers the age, height, TBW, and the 16 constituents listed in Table $1^{16}$. 
Table 1

Patient characteristics and parameters included in BIA

\begin{tabular}{|l|l|}
\hline Age (years) & $66.8 \pm 11.8$ \\
\hline Height $(\mathrm{cm})$ & $161.7 \pm 9.1$ \\
\hline Total body weight $(\mathrm{kg})$ & $64.5 \pm 11.6$ \\
\hline Total body water $(\mathrm{I})$ & $33.2 \pm 6.6$ \\
\hline Protein $(\mathrm{kg})$ & $8.8 \pm 1.8$ \\
\hline Minerals $(\mathrm{kg})$ & $3.1 \pm 0.6$ \\
\hline Body fat mass $(\mathrm{kg})$ & $19.4 \pm 6.8$ \\
\hline Soft lean mass $(\mathrm{kg})$ & $42.5 \pm 8.5$ \\
\hline Lean body mass $(\mathrm{kg})$ & $45.1 \pm 8.9$ \\
\hline Body mass index $\left(\mathrm{kg} / \mathrm{m}^{2}\right)$ & $24.6 \pm 3.4$ \\
\hline Trunk of the lean body mass $(\mathrm{kg})$ & $20.3 \pm 4.3$ \\
\hline Ratio of extracellular- to total body water & $0.4 \pm 0.1$ \\
\hline Intracellular water $(\mathrm{I})$ & $20.3 \pm 4.3$ \\
\hline Extracellular water $(\mathrm{l})$ & $12.9 \pm 2.4$ \\
\hline Skeletal muscle mass $(\mathrm{kg})$ & $24.5 \pm 5.4$ \\
\hline Basal metabolic mass $(\mathrm{cal})$ & $1343.1 \pm 192.1$ \\
\hline Osseous mineral mass $(\mathrm{kg})$ & $2.5 \pm 0.5$ \\
\hline Body cell mass $(\mathrm{kg})$ & $29.1 \pm 5.9$ \\
\hline Skeletal muscle index $\left(\mathrm{kg} / \mathrm{m}^{2}\right)$ & $7.0 \pm 1.1$ \\
\hline
\end{tabular}

\section{CT and injection protocol}

Using a $40 \mathrm{~mm}$ volume CT scanner (Lightspeed VCT; GE Healthcare, Milwaukee, WI), CE-DCT was performed with the patient in the supine position during a breath-hold. We scanned craniocaudally from the top of the liver to the lower end of the kidney. The scanning parameters were 0.5 -sec rotation scan, 5.0-mm detector row width, 0.516 helical pitch (beam pitch), 41.2-mm table movement, $50-\mathrm{cm}$ scan field of view, $120 \mathrm{kVp}$, and 100-770 mA with the automatic tube current modulation (noise index, 12). Depending on the geometry, the scanning time varied from 5 to $10 \mathrm{sec}^{10}$.

For 30-sec CM delivery with a power injector (Dual Shot; Nemoto-Kyorindo, Tokyo, Japan) we inserted a 20 -gauge catheter into an antecubital vein (600 mgl/kg, Omnipaque-300; Daiichi-Sankyo, Tokyo, Japan). Unenhanced CT images and scans obtained during the hepatic arterial- and the portal venous phase 
(HAP, PVP) were inspected; to scan during the equilibrium phase (EP) we applied a 180-sec delay. For HAP scanning we used a computer-assisted bolus tracking technique to synchronize the arrival of the $\mathrm{CM}$ at the abdominal aorta at the level of the celiac artery with the start of scanning. To monitor the arrival of the $\mathrm{CM}$, we performed axial scans of the abdominal aorta at the celiac artery level $20 \mathrm{sec}$ after the start of contrast injection. Scanning started automatically $15 \mathrm{sec}$ after contrast enhancement reached $100 \mathrm{HU}$ in a region of interest within the abdominal aorta. For PVP scanning we started the scan 20 seconds after the HAP ${ }^{10}$.

\section{Contrast enhancement in the abdominal aorta and hepatic parenchyma}

In all patients we measured the CT number in the abdominal aorta at the celiac artery level on unenhanced scans and during HAP. We also measured the mean CT number in the hepatic parenchyma of the right- and left hepatic lobe at the celiac artery level on unenhanced and PVP scans ${ }^{10}$. The CT number was recorded as the pixel value within an approximately $10 \mathrm{~mm}^{2}$ circular region of interest. Contrast enhancement was expressed as the change in the CT number calculated by subtracting the HU on unenhanced images from the HU recorded during the HAP and PVP. We also calculated the change in iodine dose per contrast enhancement (IDCE) (mgl/HU) to evaluate the effect of the patient age, TBW, height, and the 16 constituents comprising the body composition on the measured values obtained by BIA.

\section{Statistical analysis}

We performed univariate linear regression analysis to determine the effect of the body composition on BIA results and on IDCE of the abdominal aorta during the HAP and on enhancement of the hepatic parenchyma during the PVP. We used the Pearson product moment correlation coefficient ( $r$ ) to assess the strength of associations involving normal data distributions as absolute values of $r$, where $0-0.19$ was recorded as a very weak-, $0.2-0.39$ as a weak-, $0.40-0.59$ as a moderate-, $0.6-0.79$ as a strong-, and $0.8-1.0$ as a very strong correlation. Variables with a $p$ value less than 0.05 were considered statistically significant. We used "R" (R, version 3.2.2; The R Project for Statistical Computing; http://www.rproject.org/).

\section{Results}

Table 1 shows the characteristics and the mean values returned by BIA of our 236 patients.

\section{Contrast enhancement and IDCE on CE-DCT}

Contrast enhancement of the abdominal aorta during the HAP was $327.3 \pm 47.6 \mathrm{HU}$; IDCE was $121.5 \pm$ $32.1 \mathrm{mgl} / \mathrm{HU}$. For the hepatic parenchyma these values were $58.1 \pm 11.7 \mathrm{HU}(\mathrm{PVP})$ and $698.7 \pm 211.1 \mathrm{mgl}$ /HU (PVP). 
Effect of body data on IDCE of the abdominal aorta on CE-DCT scans acquired during the HAP

As shown in Table 2, univariate linear regression analysis revealed a significant correlation between IDCE of the abdominal aorta and the items included in BIA (all: $p<0.01$ ). The TBW had the strongest effect on IDCE on CE-DCT scans acquired in the HAP.

Table 2. Bioelectrical impedance analysis of body factors that the affect aortic and hepatic enhancement on contrast-enhanced dynamic CT scans 


\begin{tabular}{|c|c|c|}
\hline & $\begin{array}{l}\text { lodine dose per contrast } \\
\text { enhancement on abdominal aorta }\end{array}$ & $\begin{array}{l}\text { lodine dose per contrast } \\
\text { enhancement on hepatic } \\
\text { parenchyma }\end{array}$ \\
\hline Age (years) & 0.01 & 0.23 \\
\hline Height (cm) & 0.57 & 0.46 \\
\hline Total body weight (kg) & 0.83 & 0.69 \\
\hline Total body water (I) & 0.73 & 0.63 \\
\hline Protein $(\mathrm{kg})$ & 0.72 & 0.63 \\
\hline Minerals (kg) & 0.71 & 0.62 \\
\hline Body fat mass (kg) & 0.50 & 0.36 \\
\hline Soft lean mass $(\mathrm{kg})$ & 0.73 & 0.63 \\
\hline Lean body mass (kg) & 0.73 & 0.63 \\
\hline $\begin{array}{l}\text { Body mass index } \\
\left(\mathrm{kg} / \mathrm{cm}^{2}\right)\end{array}$ & 0.59 & 0.53 \\
\hline $\begin{array}{l}\text { Trunk of the lean body } \\
\text { mass }(\mathrm{kg})\end{array}$ & 0.72 & 0.64 \\
\hline $\begin{array}{l}\text { Ratio of extracellular- } \\
\text { to total body water }\end{array}$ & 0.20 & 0.26 \\
\hline Intracellular water (I) & 0.72 & 0.63 \\
\hline Extracellular water (I) & 0.74 & 0.62 \\
\hline $\begin{array}{l}\text { Skeletal muscle mass } \\
(\mathrm{kg})\end{array}$ & 0.72 & 0.63 \\
\hline $\begin{array}{l}\text { Basal metabolic mass } \\
\text { (cal) }\end{array}$ & 0.73 & 0.63 \\
\hline $\begin{array}{l}\text { Osseous mineral mass } \\
(\mathrm{kg})\end{array}$ & 0.70 & 0.56 \\
\hline Body cell mass (kg) & 0.72 & 0.63 \\
\hline $\begin{array}{l}\text { Skeletal muscle index } \\
\left(\mathrm{kg} / \mathrm{cm}^{2}\right)\end{array}$ & 0.75 & 0.69 \\
\hline
\end{tabular}

Effect of body data on IDCE of the hepatic parenchyma on CE-DCT scans acquired during the PVP

Table 2 also shows the results of univariate linear regression analysis of the correlation between IDCE of the hepatic parenchyma and the items included in BIA (all: $p<0.01$ ). The TBW and the skeletal muscle index (SMI) had the strongest effect on IDCE on CE-DCT scans acquired in the PVP. 


\section{Representative images}

Representative unenhanced CT-, HAP-, PVP-, and EP images are presented in Figs. 1 and 2.

\section{Discussion}

The human body is comprised of body water, protein, minerals, and body fat. The weight of human tissues such as muscles and somatic cells can be calculated from their relationship to the different components.

To our knowledge, this is the first clinical study to assess the effect of items included in BIA on IDCE on CE-CT scans of the aorta and hepatic parenchyma. In BIA, the body water- and the body components comprising the weight of the human body are further subdivided into their constituents and calculated.

The TBW exhibited the greatest effect on IDCE of the abdominal aorta scanned during the HAP, it also had the greatest effect on hepatic parenchymal enhancement observed during the PVP. The SMI most strongly affected enhancement of the hepatic parenchyma during the PVP. The other components addressed in BIA showed a moderate to high correlation with the TBW and SMI.

Earlier studies suggested that among assessed body parameters, the LBW and the BSA exhibited the strongest correlation with aortic and hepatic enhancement ${ }^{14,17}$. The most commonly used parameter for determining the iodine dose has been the TWB. However, in obese patients, the iodine dose may be excessive because a large proportion of their TBW is comprised of poorly perfused adipose tissue in which iodine distributes poorly ${ }^{18-21}$. In small patients, it may also be excessive.

Ours diverge from earlier findings possibly because the difference between the LBW or the BSA and the TBW was relatively small with respect to IDCE reported earlier ${ }^{14}$. Also, while the LBW and the BSA are factors that influence IDCE more strongly than the TBW in overweight and underweight patients, the weight distribution in our subjects was very narrow and there were only a few high-weight patients.

We found that during the PVP, the SMI exhibited the greatest effect on IDCE of the hepatic parenchyma. The SMI was calculated by dividing the limb skeletal muscle mass $(\mathrm{kg})$ by the square of the height $\left(\mathrm{m}^{2}\right)$. The SMI is an index for the skeletal muscle mass and it is used as a criterion for the diagnosis of sarcopenia, defined as an age-related loss in the skeletal muscle mass that results in a reduction in muscular strength and physical function ${ }^{22}$. The blood vessels in muscles contain a larger blood volume than does fat; this is thought to exert a strong effect on contrast enhancement ${ }^{23-25}$. The SMI is highly correlated with liver fibrosis and a correlation between liver fibrosis and contrast enhancement of the hepatic parenchyma has been reported ${ }^{26}$. Consequently, IDCE on CE-DCT scans may be strongly affected by the SMI.

We found that many body parameters included in BIA affect IDCE on CE-DCT images. Most exhibited a more than moderate correlation with IDCE of vessels and the hepatic parenchyma. 
The performance of machine-learning algorithms applied to medical data is high and machine learning is useful for predicting clinical outcomes ${ }^{27,28}$. Therefore, we think that the integration of parameters included in BIA into machine learning may yield more accurate predictions.

Our study has some limitations. Ours was a retrospective, single-center study and the study population was small. As the median and the range of the TBW of Japanese- is lower than of Western populations, our findings cannot be extrapolated to non-Japanese individuals. In this study we did not compare contrast enhancement with the BSA index, we did not confirm the relationship between contrast enhancement and the image quality, and we did not address the detectability of liver tumors. These issues are currently under investigation.

In conclusion, BIA showed that among the components comprising the weight of the human body, the TBW had the strongest effect on aortic and hepatic enhancement on CE-DCT scans. With respect to hepatic parenchymal contrast enhancement during the PVP; the SMI exhibited the greatest effect.

\section{Declarations}

\section{Author contributions}

TM. (Conceived and designed the analysis, Collected the data,

Contributed data or analysis tools, Performed the analysis, Wrote the paper)

TN. (Conceived and designed the analysis, Performed the

analysis, Collected the data)

YF. (Conceived and designed the analysis, Collected the data)

TS. (Conceived and designed the analysis, Collected the data)

KA. (Conceived and designed the analysis, Collected the data)

TO. (Conceived and designed the analysis, Collected the data)

SM. (Conceived and designed the analysis, Collected the data)

SA. (Conceived and designed the analysis, Collected the data)

$\mathrm{JH}$. (Conceived and designed the analysis, Collected the data)

KA. (Conceived and designed the analysis, Collected the data,

Contributed data or analysis tools, Wrote the paper)" 


\section{References}

1. Bonaldi, V. M., Bret, P. M., Reinhold, C. \& Atri, M. Helical CT of the liver: value of an early hepatic arterial phase. Radiology, 197 (2), 357-363 (1995).

2. Mitsuzaki, K. et al. Multiple-phase helical CT of the liver for detecting small hepatomas in patients with liver cirrhosis: contrast-injection protocol and optimal timing. AJR Am J Roentgenol, 167 (3), 753-757 (1996).

3. Van Hoe, L. et al. Dual-phase helical CT of the liver: value of an early-phase acquisition in the differential diagnosis of noncystic focal lesions. AJR Am J Roentgenol, 168 (5), 1185-1192 (1997).

4. Lee, H. M. et al. Hepatic lesion characterization in cirrhosis: significance of arterial hypervascularity on dual-phase helical CT. AJR Am J Roentgenol, 169 (1), 125-130 (1997).

5. Yanaga, Y. et al. Optimal dose and injection duration (injection rate) of contrast material for depiction of hypervascular hepatocellular carcinomas by multidetector CT. Radiat Med, 25 (6), 278-288 (2007).

6. Heiken, J. P. et al. Dynamic incremental CT: effect of volume and concentration of contrast material and patient weight on hepatic enhancement. Radiology, 195 (2), 353-357 (1995).

7. Honda, H. et al. Hepatocellular carcinoma: correlation of CT, angiographic, and histopathologic findings. Radiology, 189 (3), 857-862 (1993).

8. Murakami, T. et al. Evaluation of optimal timing of arterial phase imaging for the detection of hypervascular hepatocellular carcinoma by using triple arterial phase imaging with multidetector-row helical computed tomography. Invest Radiol, 38 (8), 497-503 (2003).

9. Masuda, T. et al. CT Angiography of Suspected Peripheral Artery Disease: Comparison of Contrast Enhancement in the Lower Extremities of Patients Undergoing and Those Not Undergoing Hemodialysis. AJR Am J Roentgenol, 208 (5), 1127-1133 (2017).

10. Masuda, T. et al. Aortic and Hepatic Contrast Enhancement During Hepatic-Arterial and Portal Venous Phase Computed Tomography Scanning: Multivariate Linear Regression Analysis Using Age, Sex, Total Body Weight, Height, and Cardiac Output. J Comput Assist Tomogr, 41 (2), 309-314 (2017).

11. Yanaga, Y. et al. Optimal contrast dose for depiction of hypervascular hepatocellular carcinoma at dynamic CT using 64-MDCT. AJR Am J Roentgenol, 190 (4), 1003-1009 (2008).

12. Kidoh, M. et al. Contrast enhancement during hepatic computed tomography: effect of total body weight, height, body mass index, blood volume, lean body weight, and body surface area. $J$ Comput Assist Tomogr, 37 (2), 159-164 (2013).

13. Onishi, H. et al. Abdominal multi-detector row CT: effectiveness of determining contrast medium dose on basis of body surface area. Eur J Radiol, 80 (3), 643-647 (2011).

14. Awai, K. et al. The Optimal Body Size Index with Which to Determine lodine Dose for Hepatic Dynamic CT: A Prospective Multicenter Study. Radiology, 278 (3), 773-781 (2016). 
15. Cha, K., Chertow, G. M., Gonzalez, J., Lazarus, J. M. \& Wilmore, D. W. Multifrequency bioelectrical impedance estimates the distribution of body water. J App/ Physiol (1985), 79 (4), 1316-1319 (1995).

16. Gibson, A. L., Holmes, J. C., Desautels, R. L., Edmonds, L. B. \& Nuudi, L. Ability of new octapolar bioimpedance spectroscopy analyzers to predict 4-component-model percentage body fat in Hispanic, black, and white adults. Am J Clin Nutr, 87 (2), 332-338 (2008).

17. Koiwahara, G. et al. Different enhancement of the hepatic parenchyma in dynamic CT for patients with normal liver and chronic liver diseases and with the dose of contrast medium based on body surface area. Jpn J Radiol, 33 (4), 194-200 (2015).

18. Bae, K. T., Heiken, J. P. \& Brink, J. A. Aortic and hepatic contrast medium enhancement at CT. Part I. Prediction with a computer model. Radiology, 207 (3), 647-655 (1998).

19. Yamashita, Y. et al. Abdominal helical CT: evaluation of optimal doses of intravenous contrast material-a prospective randomized study. Radiology, 216 (3), 718-723 (2000).

20. Awai, K. \& Hori, S. Effect of contrast injection protocol with dose tailored to patient weight and fixed injection duration on aortic and hepatic enhancement at multidetector-row helical CT. Eur Radiol, 13 (9), 2155-2160 (2003).

21. Yanaga, Y. et al. Effect of contrast injection protocols with dose adjusted to the estimated lean patient body weight on aortic enhancement at CT angiography. AJR American journal of roentgenology, $192(4), 1071-1078$ (2009).

22. Moon, J. J., Park, S. G., Ryu, S. M. \& Park, C. H. New Skeletal Muscle Mass Index in Diagnosis of Sarcopenia. Journal of bone metabolism, 25 (1), 15-21 (2018).

23. Yoshimura, K., Eto, H., Kato, H., Doi, K. \& Aoi, N. In vivo manipulation of stem cells for adipose tissue repair/reconstruction. Regenerative medicine, 6 (6 Suppl), 33-41 (2011).

24. Rowe, G. C. et al. PGC-1a induces SPP1 to activate macrophages and orchestrate functional angiogenesis in skeletal muscle. Circulation research, 115 (5), 504-517 (2014).

25. Waters, R. E., Rotevatn, S., Li, P., Annex, B. H. \& Yan, Z. Voluntary running induces fiber type-specific angiogenesis in mouse skeletal muscle. American journal of physiology Cell physiology, 287 (5), C1342-1348 (2004).

26. Hyun Kim, K. et al. Sarcopenia assessed using bioimpedance analysis is associated independently with significant liver fibrosis in patients with chronic liver diseases. European journal of gastroenterology \& hepatology, 32 (1), 58-65 (2020).

27. Heo, J. et al. Machine Learning-Based Model for Prediction of Outcomes in Acute Stroke. Stroke, 50 (5), 1263-1265 (2019).

28. Masuda, T. et al. Machine-learning integration of CT histogram analysis to evaluate the composition of atherosclerotic plaques: Validation with IB-IVUS. Journal of cardiovascular computed tomography, 13 (2), 163-169 (2019).

\section{Figures}




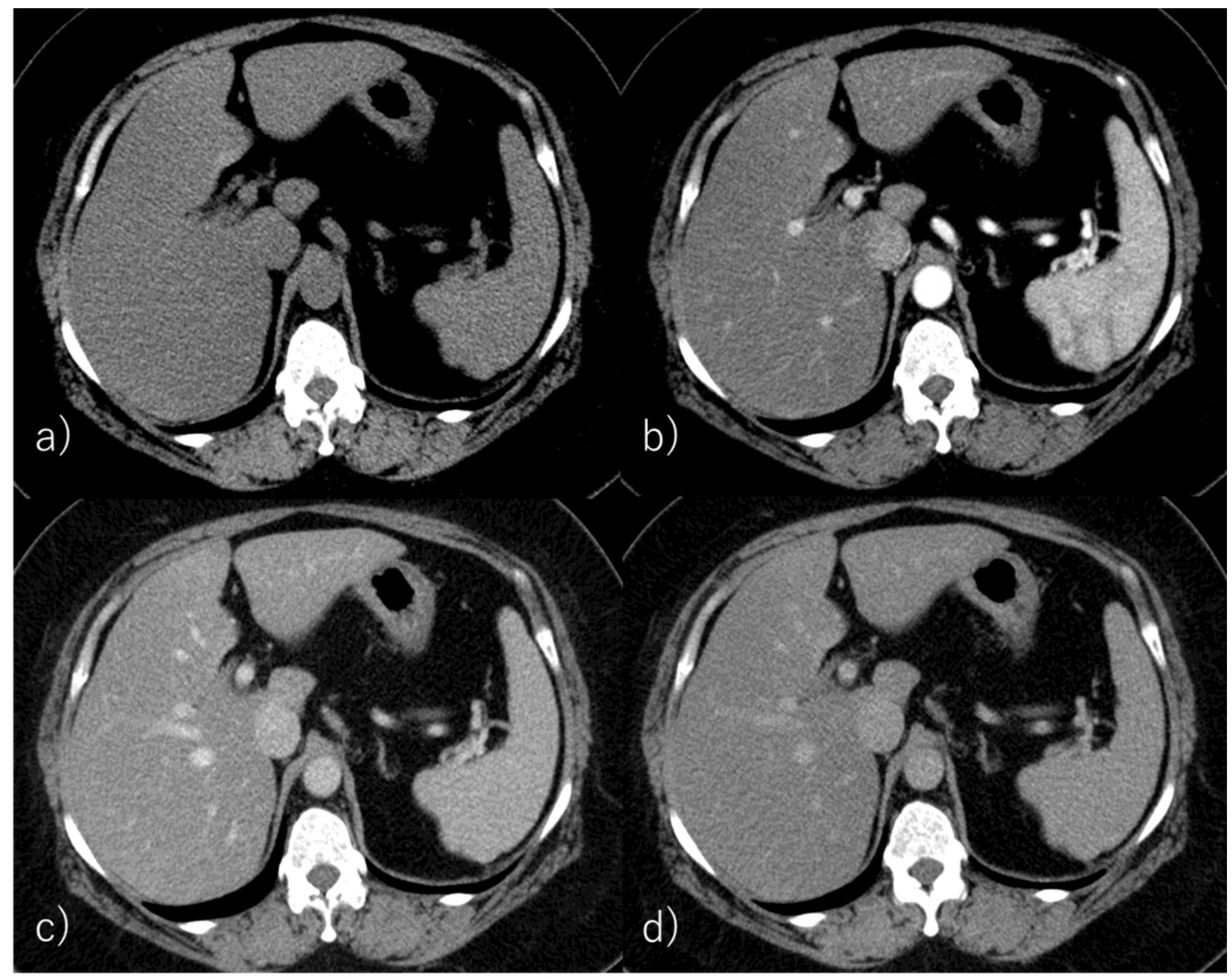

Figure 1

CE-CT images obtained in a 49-year-old woman with liver cirrhosis. Her height was $157 \mathrm{~cm}$ and her total body weight was $115 \mathrm{~kg}$. a) Unenhanced axial image b) Hepatic arterial phase c) Portal venous phase d) Equilibrium phase Her skeletal muscle index was high $(9.4 \mathrm{~kg} / \mathrm{m} 2)$ and contrast enhancement during the portal venous phase was low $(63 \mathrm{HU})$. 


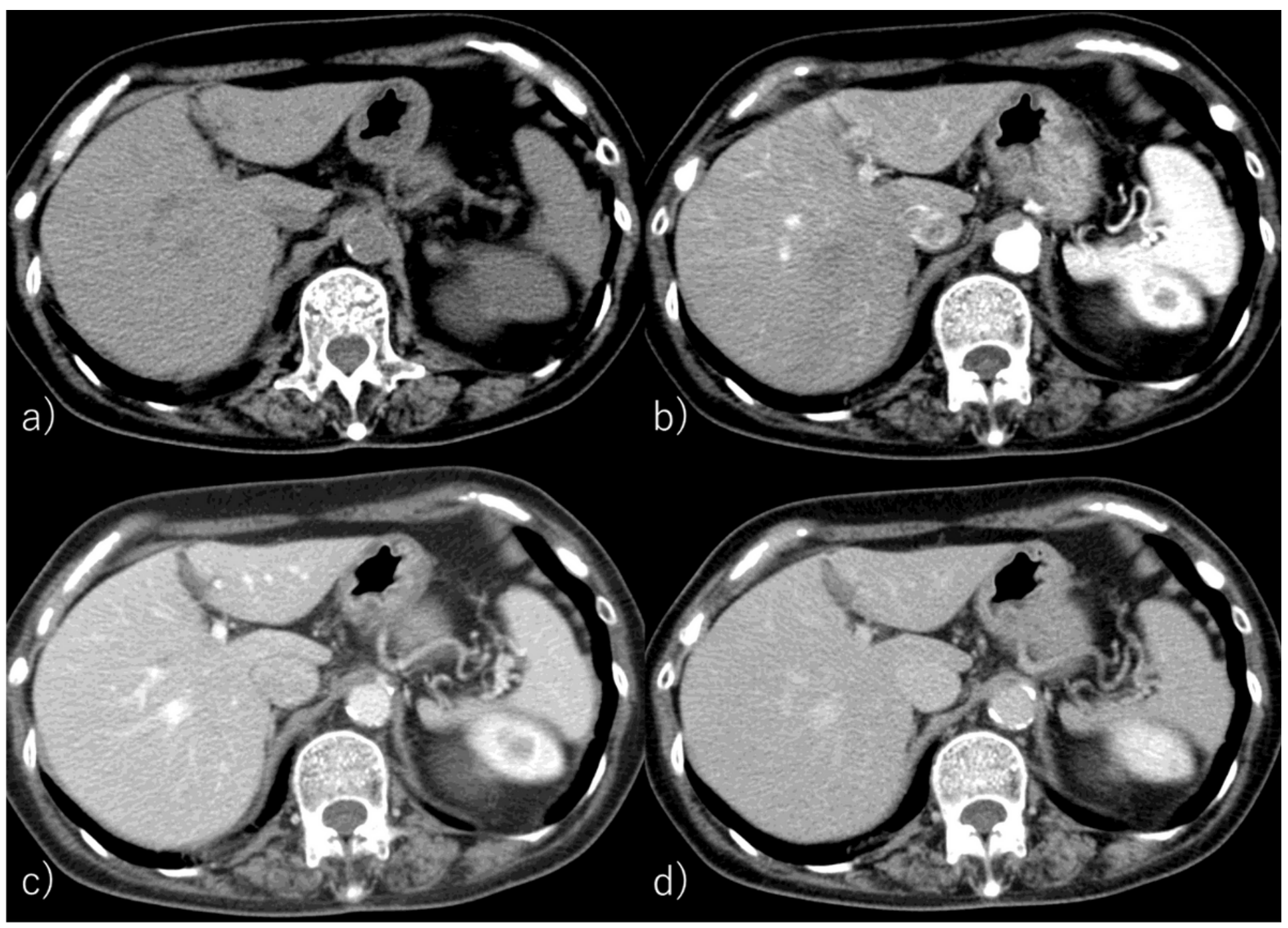

Figure 2

CE-CT images obtained in an 80-year-old woman with liver cirrhosis. Her height was $155 \mathrm{~cm}$ and her total body weight was $46 \mathrm{~kg}$. a) Unenhanced axial image b) Hepatic arterial phase c) Portal venous phase d) Equilibrium phase Her skeletal muscle index was low $(4.8 \mathrm{~kg} / \mathrm{m} 2)$ and contrast enhancement during the portal venous phase was high $(140 \mathrm{HU})$. 